\title{
Airway inflammation in symptomatic and asymptomatic children with methacholine hyperresponsiveness
}

\section{To the Editor:}

I read, with great interest, the paper by Pin and coworkers in the October issue of the Journal (1993; 6: 1249-1256). In a small sample of symptomatic and asymptomatic children with methacholine hyperresponsiveness, these workers showed that inflammatory changes may be present in the airways of asymptomatic hyperresponders, albeit to a lesser degree than in the airways of symptomatic hyperresponders. This suggests that such children may have asthma but do not seek medical attention for it. I feel, however, that a number of interesting studies regarding the same subject have not been addressed in the discussion.

Firstly, we have recently shown that asymptomatic adult hyperresponders are more likely to be poor perceivers of induced airway obstruction than are symptomatic hyperresponders [1]. This suggests that they may have (variable) airway obstruction but do not perceive this as dyspnoea. This is in line with the results of Pin and co-workers in children.

Secondly, two studies have prospectively followed-up children with asymptomatic hyperresponsiveness to establish its role as a putative risk factor for the development of clinical asthma. PEAT et al. [2] found that the majority of children with asymptomatic hyperresponsiveness were no longer hyperresponsive at followup 3 yrs later. We have recently confirmed this finding

\section{REPLY}

From the authors:

We are grateful for the opportunity to respond to the letter of Dr Brand. Our study was to examine the mechanisms of methacholine airway hyperresponsiveness in young subjects, aged 11-16 yrs. We believe that the results are highly instructive and wish to avoid misunderstanding. The subjects with no symptoms and positive methacholine tests did not have sputum eosinophilia. This finding should be noted, since this may be a reason why they were asymptomatic.

Dr Brand also suggested that our results were in line with his observation of poor perception (poor recognition of airway constriction or obstruction) in adults with asymptomatic airway hyperresponsiveness. In fact, they were not. We actually said that seven of the asymptomatic children had failed in the past to recognize airway constriction. We actually measured perception during the methacholine test, and this was not significantly different in the asymptomatic and the in our study of a population sample of schoolchildren after 27 yrs of follow-up: asymptomatic hyperresponsiveness in childhood was not a risk factor for respiratory symptoms in young adulthood [3].

Thus, although asymptomatic hyperresponders may have a mild asthmatic inflammatory reaction in their airways, it is far from clear that this has any clinical relevance. Clearly, more prospective follow-up studies of such subjects are needed.

\section{P.L.P. Brand}

Dept of Paediatrics, St Elisabeth Hospital, Breedestraat 193 (O), Curaçao, Netherlands Antilles.

\section{References}

1. Brand PLP, Rijcken B, Schouten JP, Koëter GH, Weiss ST, Postma DE. Perception of airway obstruction in a random population sample: relationship to airway hyperresponsiveness in the absence of respiratory symptoms. Am Rev Respir Dis 1992; 146: 396-401.

2. Peat JK, Salome CM, CS, Kerrebijn J, Woolcock AJ. A prospective study of bronchial hyperresonsiveness and respiratory symptoms in a population of Australian school children. Clin Exp Allergy 1989; 19: 299-306.

3. de Gooijer A, Brand PLP, Gerritsen J, Koëter GH, Postma DS, Knol K. Changes in respiratory symptoms and airway hyperresponsiveness after 27 years in a populationbased sample of school children. Eur Respir J 1993; 6: 848-854.

symptomatic children. We maintain that the lack of recognized symptoms does distinguish this group, and neither the results of the methacholine test nor sensation during the methacholine test change this fact.

Dr Brand's point about prognosis is an interesting one, but is not relevant to our study. We have expressed the view that there are several theoretical scenarios leading to asymptomatic hyperresponsiveness. It is through an investigation of the underlying pathophysiology and the measurable correlates of disease that we will come to understand differences in clinical manifestations, including prognosis.

Finally, Dr Brand states that it is far from clear that there is any clinical relevance to asymptomatic hyperesponsiveness. We are delighted for the affected children if the progress is good. However, we would interpret any findings that are correlates of a lack of symptoms or a good prognosis as having clinical significance.

\section{Pin, F.E. Hargreave, J. Dolovich}

Pneumologie Infantile, Médecine Infantile A, Centre Hospitalier Universitaire de Grenoble, BP 217, 38043 Grenoble Cedex 19, France 